\title{
Regulación de la Agresividad Entre Preescolares Mediante el Entrenamiento a Madres y Profesoras*
}

\section{Regulation of The Aggressiveness Among Pre-School Children by The Training of Mothers and Teachers}

\author{
Cristóbal Guerra \\ Universidad Santo Tomás, Chile \\ Verónica Fredes \\ Universidad del Mar, Chile
}

\author{
María de los Ángeles Campaña \\ Universidad del Mar, Chile \\ Lisette Gutiérrez \\ Universidad del Mar, Chile
}

$\&$

\author{
Hugo Plaza \\ Universidad del Mar, Chile
}

(Rec: 20 de Enero de 2010 / Acep: 14 de Septiembre de 2011)

\begin{abstract}
Resumen
El objetivo de la investigación fue evaluar la efectividad de una intervención dirigida a disminuir la frecuencia de conductas agresivas en preescolares mediante el entrenamiento a madres y profesoras. Se diseñó e implementó un programa de entrenamiento a madres y profesoras tendiente a modificar sus creencias irracionales referidas a la crianza de los niños y a capacitarlas en una serie de procedimientos de manejo conductual infantil. El diseño empleado para evaluar la efectividad del tratamiento fue el de línea base múltiple. Se analizan los resultados de la intervención en base a criterios de significación clínica y estadística. La intervención fue exitosa ya que las madres y profesoras disminuyeron sus creencias irracionales y mejoraron sus habilidades de manejo conductual para hacer frente a la agresividad de los niños. De esta manera los niños disminuyeron la frecuencia del comportamiento agresivo verbal, físico y oposicionista tanto en el contexto familiar como escolar.
\end{abstract}

Palabras clave: Agresividad, preescolares, entrenamiento a padres, intervención en contexto escolar, tratamientos efectivos.

\begin{abstract}
The aim of this research was to evaluate the effectiveness of an intervention directed to reduce the frequency of aggressive behavior among pre- school children, by training their mothers and teachers. A training program for mothers and teachers was designed and implemented, in order to modify their irrational beliefs referred to the guidance of kids and to teach them appropriate procedures for modification in the children's behavior. The design for the assessment of an effective treatment implementation was the so called multiple baseline design in terms of clinical and statistical meaning. The intervention was successful since the mothers and teachers diminished his irrational beliefs and improved their skills of behavior modification when tackling the aggressiveness of the toddlers. On the other hand, the children also diminished the frequency of aggressive verbal, physical and opponent behavior both within the family and school contexts.
\end{abstract}

Key words: Aggressiveness, pre- school children, parents' training, intervention in school context, effective treatments. 


\section{Introducción}

La agresividad entre escolares es un fenómeno de creciente interés internacional debido a su alta incidencia en todo el mundo (Trautmann, 2008). Esto se ve reflejado en distintos estudios de prevalencia y en estudios que buscan delimitar sus consecuencias.

En Chile el 45,5\% de los estudiantes de colegios públicos han observado episodios de violencia física entre sus pares (Zerón, 2002), mientras que en España el 48\% de los estudiantes de secundaria han sido agredidos por sus pares (Gutiérrez, Barrios; de Dios, Montero \& del Barrio, 2008). Por su parte, un estudio de prevalencia realizado en 25 países revela que la agresividad escolar varía entre un $9 \%$ en Suecia y un $54 \%$ en Lituania (Nansel, Craig, Overpeck, Saluda \& Ruan, 2004).

Respecto a las consecuencias de la agresividad entre escolares, se ha documentado que un comportamiento excesivamente agresivo en la infancia se asocia al fracaso académico (Lozano \& García, 2000), predice la agresividad durante la adolescencia y la edad adulta (Kellam, Rebok, Ialongo \& Mayer, 1994) y predice otras patologías psicológicas durante la edad adulta (De la Barra, Toledo \& Rodríguez, 2003). Por otro lado, los trastornos depresivos y ansiosos, el ausentismo escolar, el deterioro en el rendimiento académico y los desajustes psicosociales en la adultez son indicadores comúnmente visibles en las víctimas de las agresiones (Trautmann, 2008).

La psicología ha aportado en la generación de intervenciones destinadas a disminuir la agresividad entre escolares y a tratar sus consecuencias. En esta línea, Trianes \& Fernández-Figarés (2001) desarrollaron un programa de intervención tendiente a la mejora del clima escolar en adolescentes mediante el entrenamiento en estrategias de resolución de conflictos. Además hay una serie de autores que han implementado, con éxito, programas de modificación conductual para regular las conductas disruptivas y agresivas en escolares. Por ejemplo, Aguilar \& Navarro (2008) utilizaron la economía de fichas y el contrato de contingencias en alumnos de primaria; Pérez, Fernández, Rodríguez \& De la Barra (2005) aplicaron un programa de contingencias llamado "el juego del buen comportamiento", también en niños de primaria; Livacic-Rojas, Espinoza \& Ugalde (2004) aplicaron un programa de habilidades sociales a adolescentes; mientras que Corsi, Barrera, Flores, Perivancich \& Guerra (2009) utilizaron la economía de fichas, la alabanza verbal, las instrucciones y el costo de respuesta en estudiantes de secundaria.

Todos los trabajos recién descritos han sido dirigidos a escolares de distintas edades, pero ninguno de ellos aborda el fenómeno de la agresividad en preescolares. Esto es inquietante ya que se han descrito indicadores del comportamiento antisocial y de agresividad injustificada a partir de los 3 años, por lo que se hace necesario realizar investigaciones con esta población (Benítez \& Justicia, 2006; Ortega \& Monks, 2005).

Motivados por lo anterior, y considerando que hoy en día el ingreso al sistema educativo en muchos países comienza en el jardín de infantes, es que ésta investigación pretendió diseñar, implementar y evaluar una intervención tendiente a la disminución de la frecuencia de la conducta agresiva en preescolares. Además, las intervenciones en la etapa preescolar generan un alto impacto ya que, como señalan Urzúa, Ramos, Alday \& Alquinta (2010), los preescolares son altamente suceptibles a la influencia ambiental debido al proceso de maduración neuropsicológica.

La intervención se sustenta en el entrenamiento a madres y profesoras ya que está documentada la relación entre los estilos de crianza, caracterizados por la difusión de normas y por la utilización excesiva del castigo, con la conducta desadaptativa en niños (Benítez \& Justicia, 2006; Mestre, Tur, Samper, Nácher \& Cortés, 2007; Schwartz, Dodge, Pettit \& Bates, 1997). Por otro lado, diversos estudios reportan resultados beneficiosos de realizar intervenciones que incluyan a padres (Corsi, Guerra \& Plaza, 2007; Rey, 2006) y a profesores (Corsi, Barrera, Flores, Perivancich, \& Guerra 2009, Trautmann, 2008).

\section{Método}

\section{Participantes}

Participaron de este estudio un grupo de 3 preescolares varones, con sus respectivas madres y profesoras.

El rango de edad de los niños fluctuó entre los 3 años con 6 meses y los 4 años con 6 meses. Todos los niños pertenecían a familias de bajos recursos que residían y estudiaban en la ciudad de Quilpué, en Chile. Los tres niños presentaban una alta frecuencia de conductas agresivas tanto en su casa como en el contexto escolar. Específicamente presentaban una alta frecuencia de conducta agresiva verbal (gritos, burlas, garabatos e insultos hacia sus pares y adultos), conducta agresiva física (golpes de puño y pie, mordiscos, lanzamiento de objetos hacia sus pares y adultos) y oposicionismo (lanzar objetos al suelo, ejecutar pataletas, prohibir acciones a otros y negarse a seguir las instrucciones dadas por madres y profesoras).

El rango de edad de las madres y profesoras fluctuó entre los 22 y 48 años. Tanto madres como profesoras presentaban una serie de creencias irracionales referidas a la crianza de los niños (ej. "nada de lo que haga va a hacer cambiar a este niño", "si no dejo que el niño haga lo que quiera seré una mala madre/profesora"). Además presentaban dificultades para abordar las conductas agresivas de los niños (instrucciones poco claras, refuerzo de conductas agresivas, ausencia de refuerzo ante las conductas positivas de los niños, inconsistencia en la aplicación de normas, maltrato físico y verbal hacia los niños). 
Al finalizar el programa de intervención se esperó que las madres y profesoras disminuyeran la frecuencia de sus creencias irracionales referidas a la crianza de sus hijos o alumnos respectivamente. Además se esperó que las madres y profesoras mejoraran sus habilidades de manejo conductual, específicamente que fueran capaces de dar instrucciones de forma adecuada (asegurándose que el niño esté prestando atención, dando la instrucción en un tono firme y describiendo claramente la conducta solicitada al niño) y de administrar técnicas de refuerzo y castigo de forma adecuada (reforzando conductas prosociales y castigando las conductas agresivas de forma consistente y contingente a la emisión de la conducta por parte del niño). Finalmente, se esperó que los niños disminuyeran la frecuencia de la conducta agresiva verbal, física y oposicionista.

\section{Instrumentos}

1. Escala de creencias irracionales para madres y profesoras (Campaña, Fredes \& Gutiérrez, 2008). Corresponde a una escala de autorregistro de 12 ítems, divididos en 3 subescalas de 4 ítems cada una. Cada subescala mide un tipo de creencia irracional asociada a la crianza de los niños; tremendismo (ejemplos: castigar al niño es algo terrible, es imposible controlar a este niño), nosoportitis (ejemplos: no soporto ver al niño llorar cuando lo disciplino, no soporto no lograr lo que me propongo con el niño), y autocondena (ejemplos: el niño no me va a querer si le pongo reglas, castigarlo me hace una mala madre/profesora).

Las participantes debieron indicar con qué frecuencia experimentaban la creencia medida por cada ítem. La escala de frecuencias incluye los siguientes valores: $0=$ nunca, 1 $=\mathrm{a}$ veces, $2=$ siempre. Para interpretar los resultados se sumaron los puntajes de los reactivos correspondientes a cada subescala, estos puntajes van de 0 a 8 (a mayor puntaje mayor frecuencia de las creencias irracionales).

2. Hojas de registro para las conductas de niños, madres y profesoras: Se utilizaron hojas de registro de la frecuencia con que cada conducta objetivo era emitida por los participantes. Las hojas de registro estaban divididas en celdas que representaban intervalos de 5 minutos. De este modo, cada sesión de observación de 45 minutos fue representada en una hoja dividida en 9 intervalos. Se marcaba la presencia de la conducta objetivo cuando ésta se presentaba, a lo menos una vez, dentro del intervalo de tiempo correspondiente. Esta forma de registro se denomina muestreo temporal de intervalo parcial (Cooper, Heron \& Heward, 2006).

Posteriormente se calculó el porcentaje de intervalos en que estuvo presente cada conducta objetivo y se graficó su frecuencia. Para asegurar la fiabilidad de las observaciones, se utilizó el método de observadores independientes durante todas las mediciones. Los observadores independientes alcanzaron un $88 \%$ de acuerdo, lo que es adecuado según los criterios de Forehand \& Mc Mahon (1981).

\section{Diseño}

Se utilizó un diseño de línea base múltiple tanto para evaluar los cambios en las creencias de las madres y profesoras, como para evaluar los cambios en las conductas de las madres, profesores y niños (medidas en cada sesión individual). Las fases de intervención consideradas en este diseño fueron:

1. Línea Base: En esta fase se registró el puntaje obtenido en la escala de creencias irracionales por madres y profesoras previo a la implementación del tratamiento. Además se registró el porcentaje del tiempo en que madres y profesoras daban instrucciones adecuadamente a los niños y administraban contingencias adecuadamente ante la conducta de los niños, previo al inicio del tratamiento. Finalmente se registró el porcentaje del tiempo en que los niños emitían conductas agresivas verbales, físicas y oposicionistas antes de iniciado el tratamiento. Las sesiones de línea base fueron 3 para el primer niño (también para su madre y profesora), 5 para el segundo niño (también para su madre y profesora) y 7 para el tercer niño (también para su madre y profesora)

2. Fase de tratamiento: En esta fase se registró el puntaje obtenido por madres y profesoras en la escala de creencias irracionales, las conductas objetivo de madres y profesoras y las conductas objetivo de los niños, una vez iniciada la intervención. Todos los participantes recibieron 7 sesiones de tratamiento individual que se realizaron en un período de 6 semanas. Además las madres y profesoras (por separado) participaron de un taller grupal (de 3 sesiones de 2 horas cada una), realizadas en las dependencias del establecimiento educacional.

3. Fase de seguimiento: En esta fase se registraron las cogniciones y conductas objetivo de los participantes después de quince días y después de un mes del término del tratamiento.

\section{Procedimiento}

Se realizó un proceso de evaluación conductual tendiente a identificar las conductas objetivo y las variables asociadas a su mantención. Se estableció que las creencias irracionales de las madres y profesoras se asociaban a sus dificultutades en el manejo conductual de los niños. A su vez, se consideró que las conductas agresivas de los niños eran mantenidas por este manejo conductual inadecuado de madres y profesoras. En la figura 1 se presenta la relación entre las variables recién descritas.

Luego, para lograr los cambios cognitivo-conductuales en madres y profesoras se utilizaron dos modalidades de intervención: La primera fue un taller grupal dirigido a madres y profesoras. La segunda fue un conjunto de sesiones individuales con madres y profesoras, en presencia de los niños, realizadas en sus propias casas y en el colegio. 


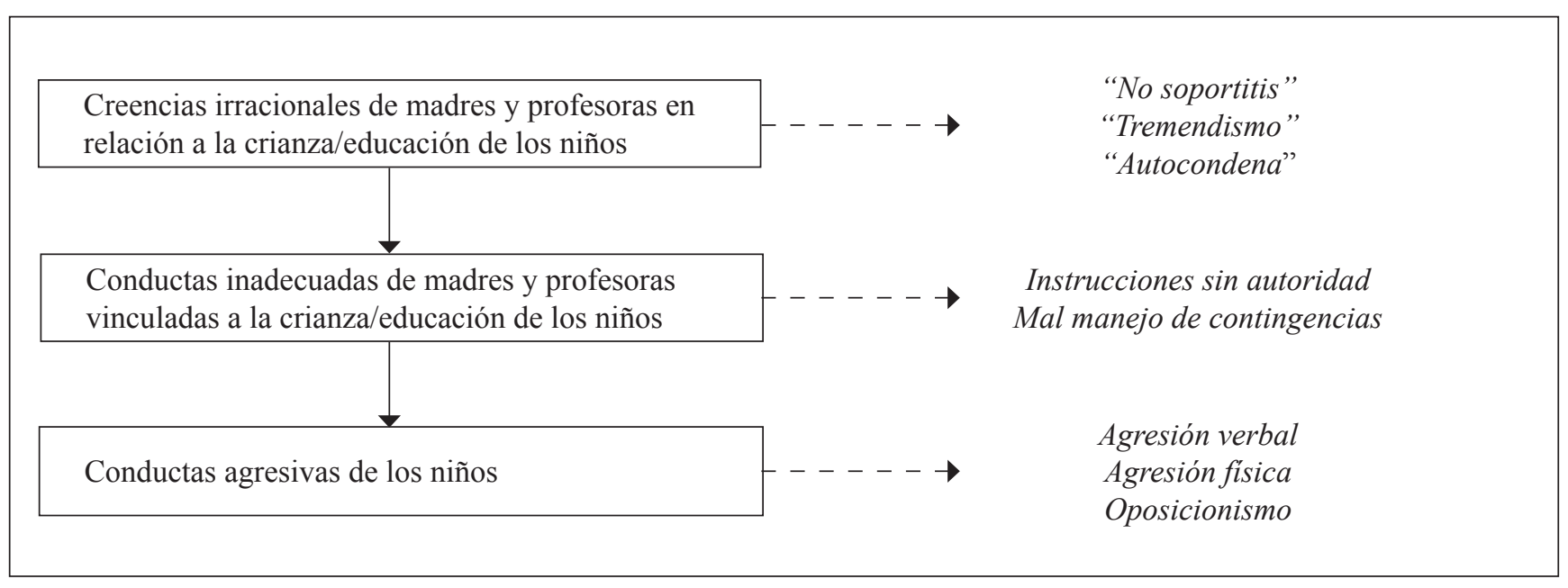

Figura 1: Evaluación conductual: a profesoras, madres y niños

Durante las sesiones grupales, madres y profesoras fueron entrenadas en técnicas de modificación conductual para aplicar con los niños y se trabajó en la modificación de sus creencias irracionales. Para modificar las creencias irracionales se utilizó autorregistro y debate cognitivo (para ver una descripción detallada de estas técnicas consultar Ellis, 1999). Luego, para capacitarlas en las técnicas de modificación conductual se utilizó la psicoeducación (donde los investigadores exponían, en términos simples, los fundamentos del análisis conductual aplicado), el modelado (mediante juego de roles los investigadores mostraban la forma de aplicar las técnicas), la imaginería (donde madres y profesoras imaginaban los efectos favorables de las técnicas a aplicar) y el entrenamiento en autoinstrucciones (de forma que madres y profesoras pudieran guiar la propia ejecución de las técnicas aprendidas). Para una descripción detallada de estas técnicas consultar Caballo (1998).

Paralelamente en las sesiones de entrenamiento individual se utilizó el ensayo de conducta, donde madres y profesoras debían aplicar directamente a los niños las técnicas de modificación conductual aprendidas en las sesiones del taller grupal. El rol de los investigadores incluyó moldear la conducta de madres y profesoras, es decir, entregar retroalimentación y refuerzos verbales a las participantes de manera de ayudarlas a mejorar gradualmente en la ejecución de las técnicas. Para una descripción detallada de estas técnicas consultar Caballo (1998).

Mediante estas dos modalidades de entrenamiento las madres y profesoras fueron entrenadas en una serie de técnicas de modificación conductual para aplicar con los niños. Las profesoras fueron entrenadas en la forma de aplicar instrucciones y contingencias adecuadas a la conducta de los niños en el contexto escolar. Específicamente las profesoras fueron entrenadas en técnicas de refuerzo verbal, economía de fichas, guía manual y costo de respuesta. Paralelamente, en el hogar de los niños, las madres fueron entrenadas en las mismas técnicas, con la diferencia que en lugar de la economía de fichas se incorporó el tiempo fuera de refuerzo (de 5 minutos) y la restricción física pasiva. Para una revisión más detallada de las técnicas aplicadas por profesoras y madres revisar Foxx, 1982a/b). La figura 2 muestra cómo se integran las distintas modalidades y contextos de la intervención.

Para evaluar la significación clínica de los cambios generados por el tratamiento se llevaron los datos tomados de las conductas y cogniciones de cada uno de los participantes, en cada una de las sesiones individuales, a un gráfico de línea base múltiple de 3 sujetos. Finalmente, para evaluar la significación estadística de los cambios generados por el tratamiento se realizaron análisis con la prueba $\mathrm{F}$ de ANOVA para cada una de las variables cognitivas y conductuales medidas. Además se evaluaron las diferencias entre medias mediante contraste pos hoc DMS, igualdad de varianza de error (contrastes de Levene), la potencia de la prueba y el tamaño del efecto. Por tratarse de un diseño de línea base múltiple, no se registró el mismo número de sesiones de línea base para todos los participantes (generando datos perdidos en las sesiones de línea base de los niños 1 y 2 , sus madres y profesoras). Para realizar los análisis estadísticos se reemplazaron los datos perdidos por la media obtenida por cada uno de estos sujetos en la fase de línea base. Para la realización de los análisis estadísticos se utilizó el programa SPSS 11.0.

\section{Resultados}

\section{Observación directa y análisis visual}

Dado que esta intervención se realizó paralelamente en el contexto escolar y familiar, los resultados se expresan de esa manera. 


\section{Técnicas de entrenamiento a profesoras}

Utilizadas en sesiones grupales:

- Psicoeducación

- Autorregistro de creencias irracionales

Debate cognitivo

Imaginería

Entrenamiento en auto-instrucciones

- Modelado
Utilizadas en la sala de clases y recreo:

- Ensayo de conducta

- Moldeamiento

\section{Efectos del entrenamiento a las profesoras}

- Disminuyen creencias irracionales (tremendismo, autocondena y nosoportitis)

- Mejoran desempeño en la entrega de instrucciones a los niños

Mejoran el desempeño en la entrega de contingencias de refuerzo y castigo a los niños (alabanza verbal, guía manual, entrega y retiro de fichas)

\section{Cambio de conductas de los niños en contexto escolar}

- Disminuyen conductas oposicionistas

- Disminuyen conductas de agresión verbal

- Disminuyen conductas de agresión física

Cambio de conductas de los niños en contexto familiar

Disminuyen creencias irracionales (tremendismo, autocondena y nosoportitis)

- Mejoran desempeño en la entrega de instrucciones a los niños

- Mejoran el desempeño en la entrega de contingencias de refuerzo y castigo a los niños (alabanza verbal, guía manual, tiempo fuera, restricción física pasiva)

\section{Efectos del entrenamiento a las madres}

\begin{tabular}{|l|l|}
\hline Utilizadas en sesiones grupales: & $\begin{array}{l}\text { Utilizadas en sesiones individuales en la casa: } \\
-\quad \text { Ensayo de conducta } \\
-\quad \text { Psicoeducación }\end{array}$ \\
- $\quad$ Autorregistro de creencias irracionales & Moldeamiento \\
- Debate cognitivo & \\
$-\quad$ Imaginería & \\
$-\quad$ Entrenamiento en auto-instrucciones & \\
\hline \multicolumn{2}{|c|}{ Técnicas de entrenamiento a las madres } \\
\hline
\end{tabular}

Figura 2: Resumen del programa de intervención 


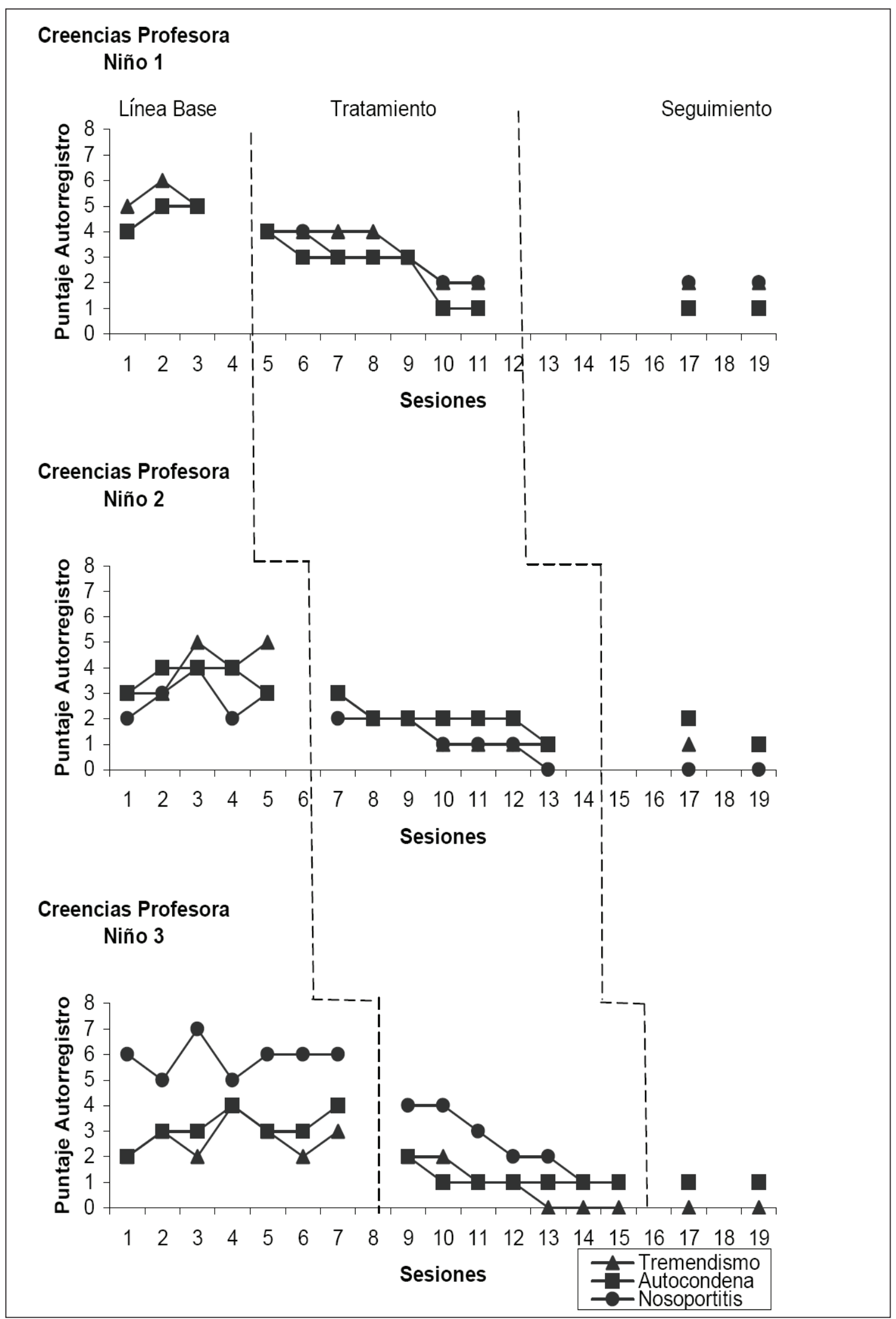

Figura 3: Cambios cognitivos en las profesoras en contexto escolar 


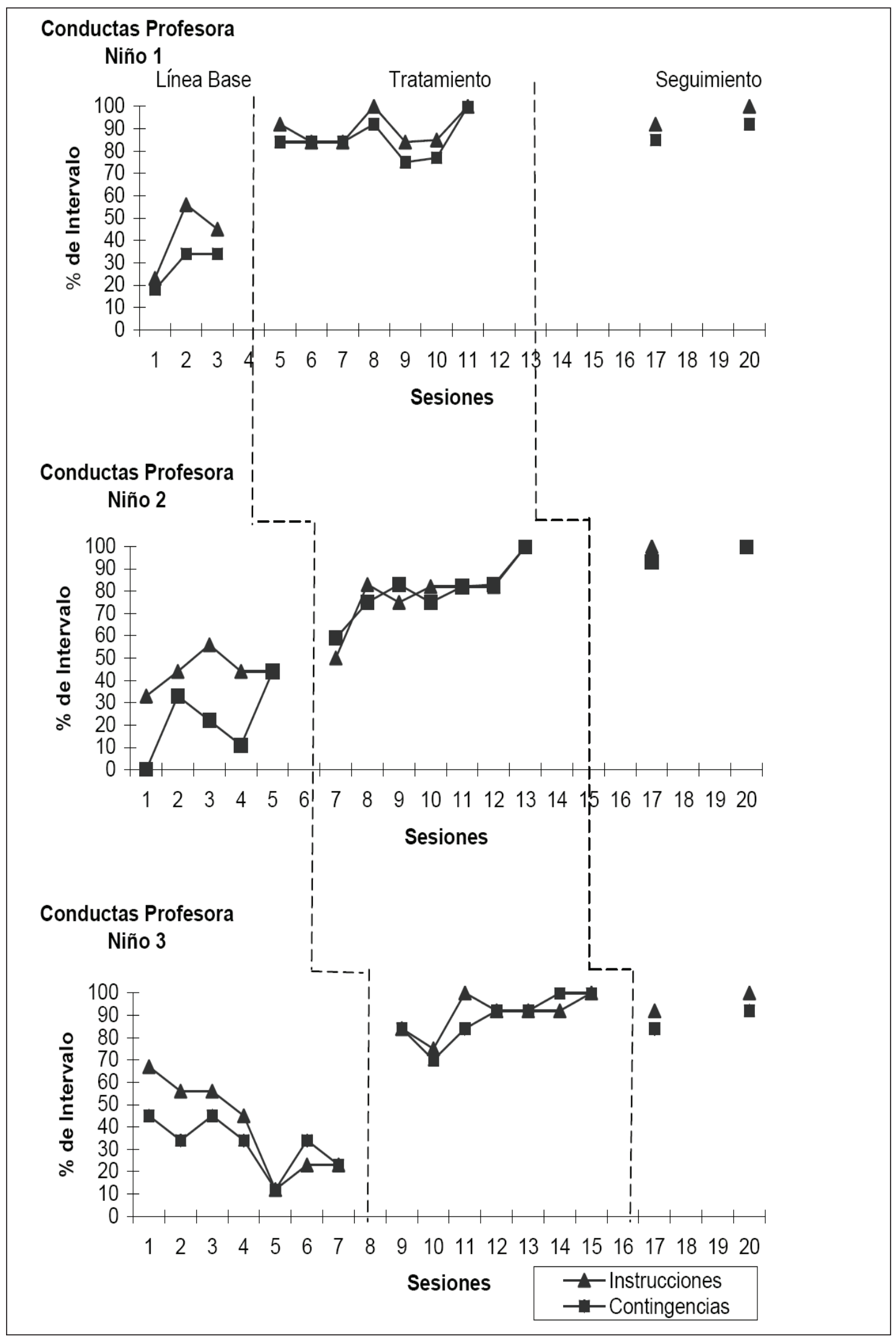

Figura 4: Cambios Conductuales en las profesoras en contexto escolar 


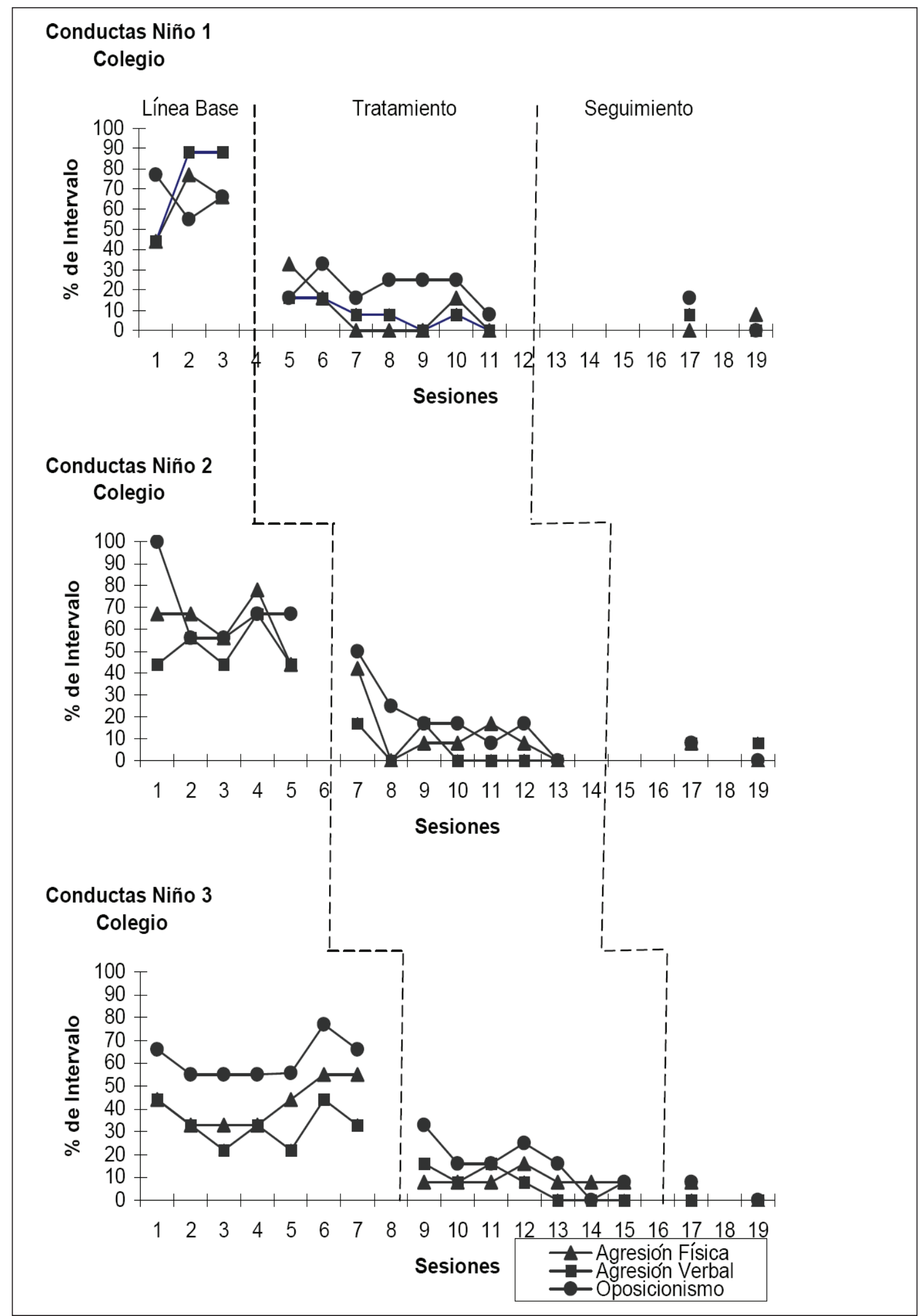

Figura 5: Cambios Conductuales en los niños en contexto escolar 
La figura 3, 4 y 5 representa los cambios cognitivos y conductuales en el contexto escolar. En la fase de línea base las tres profesoras presentaban una alta frecuencia de creencias irracionales del tipo nosoportitis (rango entre 2 y 7 puntos), tremendismo (rango entre 2 y 6 puntos) y autocondena (rango entre 2 y 5 puntos), vinculadas al comportamiento de los niños. Esto se asociaba a un bajo rendimiento en la entrega de instrucciones adecuadas a los niños (entre el 23\% y 56\% del tiempo), así como un bajo rendimiento en la aplicación adecuada de contingencias de refuerzo y castigo a los mismos (entre el $0 \%$ y $45 \%$ del tiempo). Consecuentemente, los tres niños presentaban, durante la línea base, una alta frecuencia de conducta agresiva verbal (entre el 22\% y $88 \%$ del tiempo), física (entre el 33\% y $78 \%$ del tiempo) y oposicionista (entre el $55 \%$ y $100 \%$ del tiempo). Una vez iniciada la intervención, las creencias irracionales de las tres profesoras fueron disminuyendo su frecuencia gradualmente hasta llegar a niveles mínimos (2 puntos o menos en todos los casos). La disminución de la frecuencia de las creencias irracionales de las profesoras coincidió con la mejora en su desempeño para hacer frente a la conducta de los niños. Específicamente, durante la intervención las profesoras lograron aplicar de forma adecuada instrucciones verbales a sus alumnos, así como contingencias de refuerzo y castigo adecuadas (alabanza verbal, entrega de fichas, guía manual y costo de respuesta). El rendimiento adecuado en ambas conductas objetivo llegó al 100\% del tiempo, en las tres profesoras, durante la última sesión de intervención. Tal como se esperaba, una vez que las profesoras comenzaron a mejorar su desempeño en la administración de instrucciones y contingencias, los niños presentaron cambios conductuales en el sentido deseado, al disminuir la frecuencia del comportamiento agresivo físico (entre el $0 \%$ y $8 \%$ en la última sesión de intervención), verbal (llegando al $0 \%$ en la última sesión de intervención en los tres niños) y oposicionista (entre el $0 \%$ y $8 \%$ en la última sesión de intervención). Estos resultados se mantuvieron durante la fase de seguimiento realizado a los 15 días y al mes después de finalizada la intervención, lo que da cuenta de la efectividad del tratamiento en el contexto escolar.

En el contexto familiar, la intervención también fue exitosa ya que, como se puede ver en las figura 6,7 y 8 las madres de los tres niños presentaban una alta frecuencia de las creencias irracionales medidas en línea base: nosoportitis (rango entre 2 y 8 puntos), tremendismo (rango entre 5 y 8 puntos) y autocondena (rango entre 2 y 8 puntos). Tal como en el caso de las profesoras, la existencia de creencias irracionales se asociaba al bajo rendimiento en la entrega de instrucciones (entre el $0 \%$ y $67 \%$ del tiempo) y contingencias (entre el $0 \%$ y $67 \%$ del tiempo) adecuadas a sus hijos, antes de iniciada la intervención. Consecuentemente, los tres niños presentaban, en sus casas, los mismos desajustes conductuales observados en el colegio: alta frecuencia de conducta agresiva verbal (entre el 10\% y $77 \%$ del tiempo), física (entre el $11 \%$ y $66 \%$ del tiempo) y oposicionista entre el $44 \%$ y $100 \%$ del tiempo). Durante la intervención las madres lograron disminuir gradualmente sus creencias irracionales hasta llegar a niveles mínimos en la última sesión (puntuaciones de 3 puntos o menos en todos los casos). Además las madres mejoraron el manejo conductual de sus hijos. Prueba de ello es que comenzaron a dar instrucciones y a aplicar contingencias de refuerzo y castigo de manera adecuada a sus hijos. Específicamente, aplicaron adecuadamente la alabanza verbal de conducta específica, la guía manual, el tiempo fuera de refuerzo y la restricción física pasiva. El desempeño apropiado de las madres en la entrega de instrucciones y aplicación de contingencias de refuerzo y castigo alcanzó el $100 \%$ del tiempo en la última sesión de intervención. Como era de esperarse los niños disminuyeron la frecuencia de la conducta agresiva verbal, física y oposicionista (estos tres tipos de conducta estuvieron ausentes durante la última sesión de intervención). Estos resultados se mantuvieron durante las mediciones de seguimiento realizadas a los 15 días y al mes después de finalizada la intervención.

\section{Análisis estadístico}

En el contexto escolar, se observa una disminución estadísticamente significativa en las creencias irracionales de las profesoras (tremendismo, autocondena y nosoportitis) entre las condiciones línea base y tratamiento. En el caso del tremendismo, se obtiene un valor $\mathrm{F}=316,162$ $(p=0,003)$, un tamaño del efecto observado de 0,994 , la potencia de prueba es de 1 y el contraste de varianzas de Levene sobre la igualdad de varianzas de error no es estadísticamente significativo $(p=0,427)$. A su vez, tanto para las condiciones de línea base y tratamiento existe un ajuste a la distribución normal con $\mathrm{Z}$ de Kolmogorov Smirnov de $0,354(\mathrm{p}=0,917)$ y de $0,471(\mathrm{p}=0,980)$ respectivamente. Habiéndose realizado contraste post Hoc DMS, se evidencia un valor estadísticamente significativo $(\mathrm{p}=0,003)$ a partir de la sesión 3 de tratamiento. En el caso de la autocondena de las profesoras se obtiene un valor $\mathrm{F}=19,857(\mathrm{p}=0,0001)$, tamaño del efecto de 0,908 y potencia de prueba de 0,182 . Por otro lado, el contraste de varianzas de Levene no es significativo $(p=0,577) y$, tanto para las condiciones de línea base y tratamiento existe ajuste a la distribución normal con $\mathrm{Z}$ de Kolmogorov Smirnov de 0,438 ( $\mathrm{p}=0,991)$ y de $0,380(p=0,997)$ respectivamente. El contraste post Hoc DMS indica un cambio significativo $(\mathrm{p}=0,020)$ a partir de la sesión 3 de tratamiento. En el caso de las creencias del tipo nosoportitis se obtiene un $\mathrm{F}=291,967(\mathrm{p}=0,035)$, un tamaño del efecto de 0,891 , una potencia de prueba de 0.572 , un contraste de varianzas de Levene no significativo $(\mathrm{p}=0,864)$ y un ajuste normal de las condiciones de línea base y tratamiento ( $\mathrm{Z}$ de Kolmogorov Smirnov de 0,401; $\mathrm{p}=0,996$ y 0,$438 ; \mathrm{p}=0,991$ respectivamente). Después de realizar contrastes post Hoc DMS, se evidencia una 


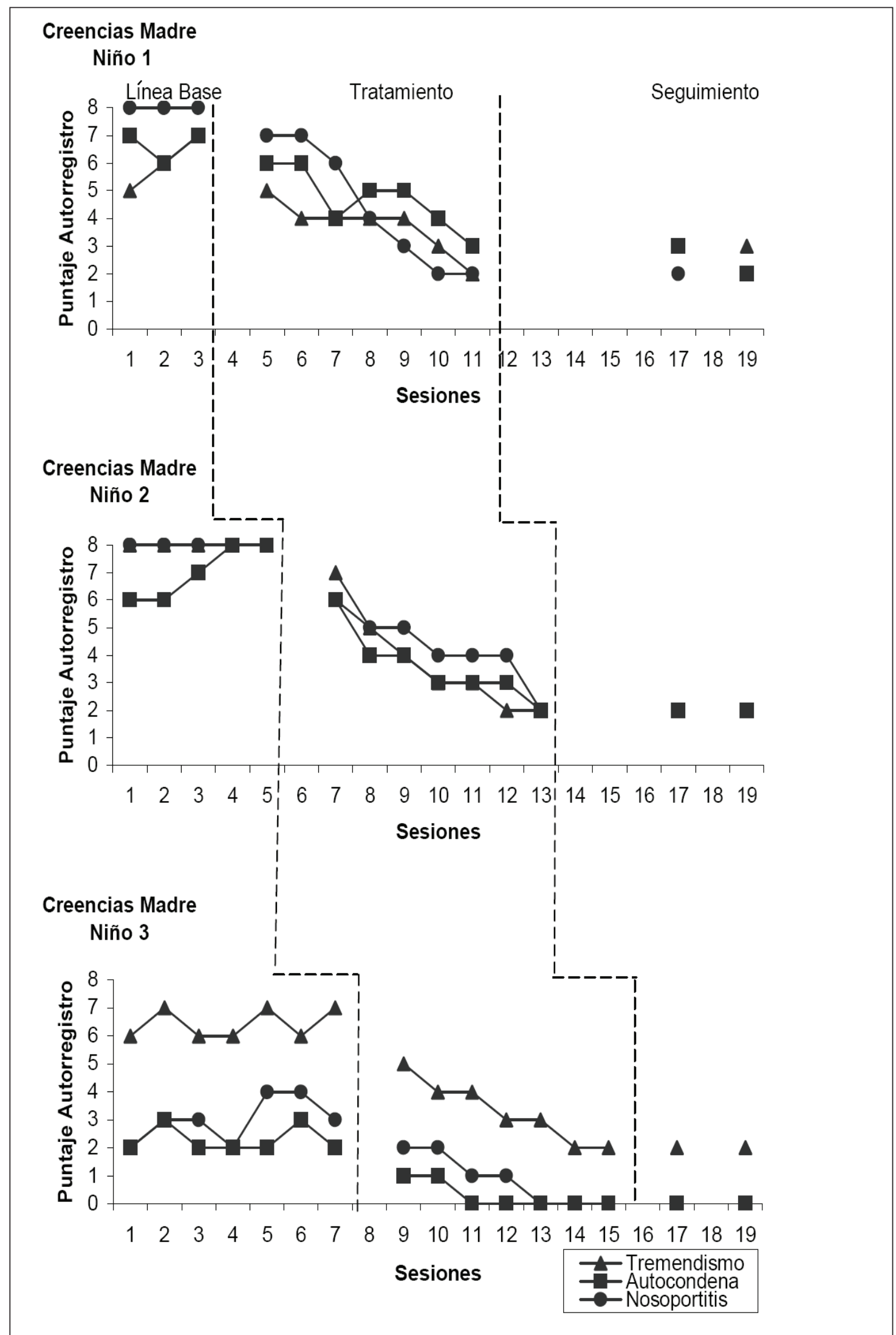

Figura 6: Cambios cognitivos en las madres en contexto familiar 


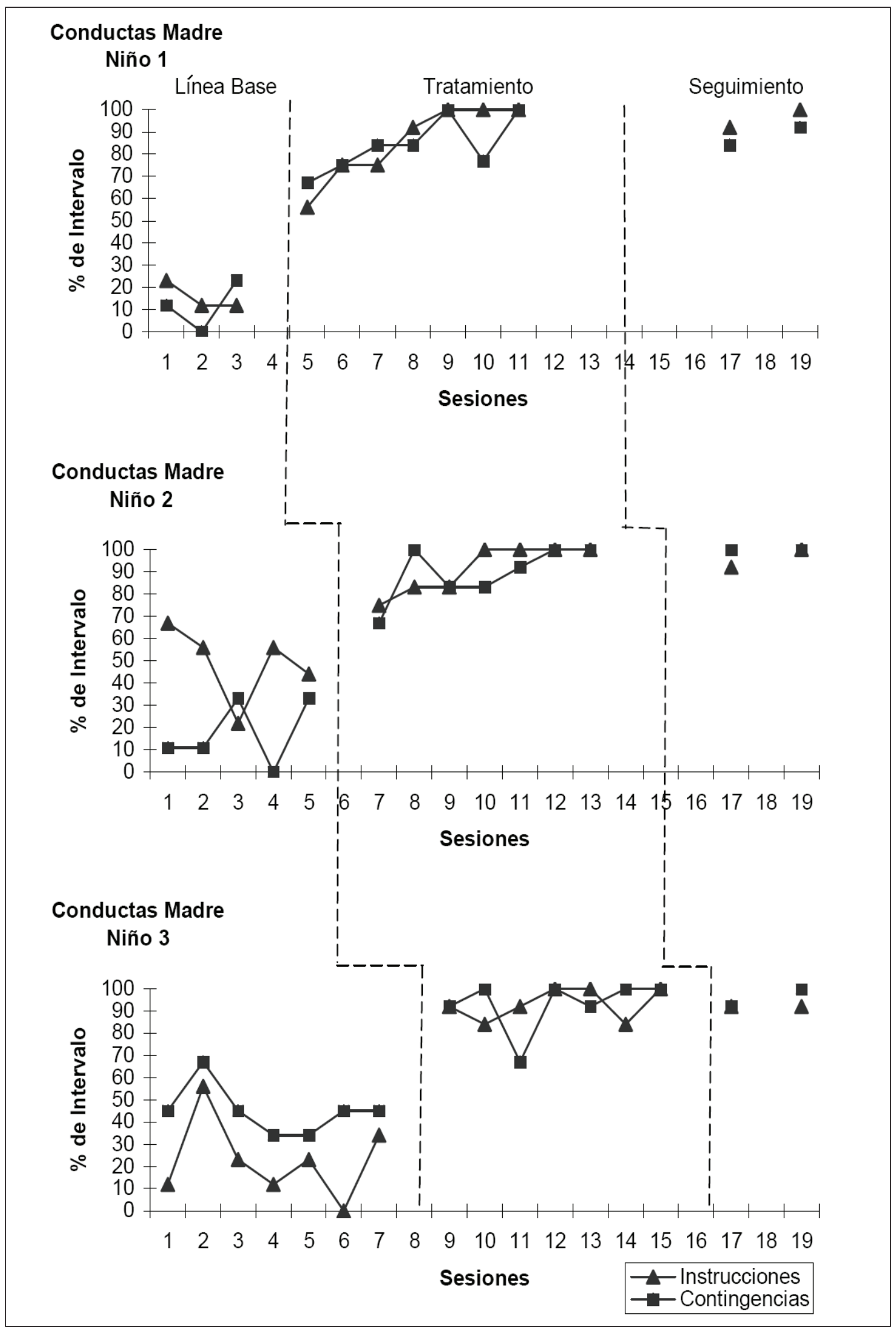

Figura 7: Cambios Conductuales en las madres en contexto familiar 


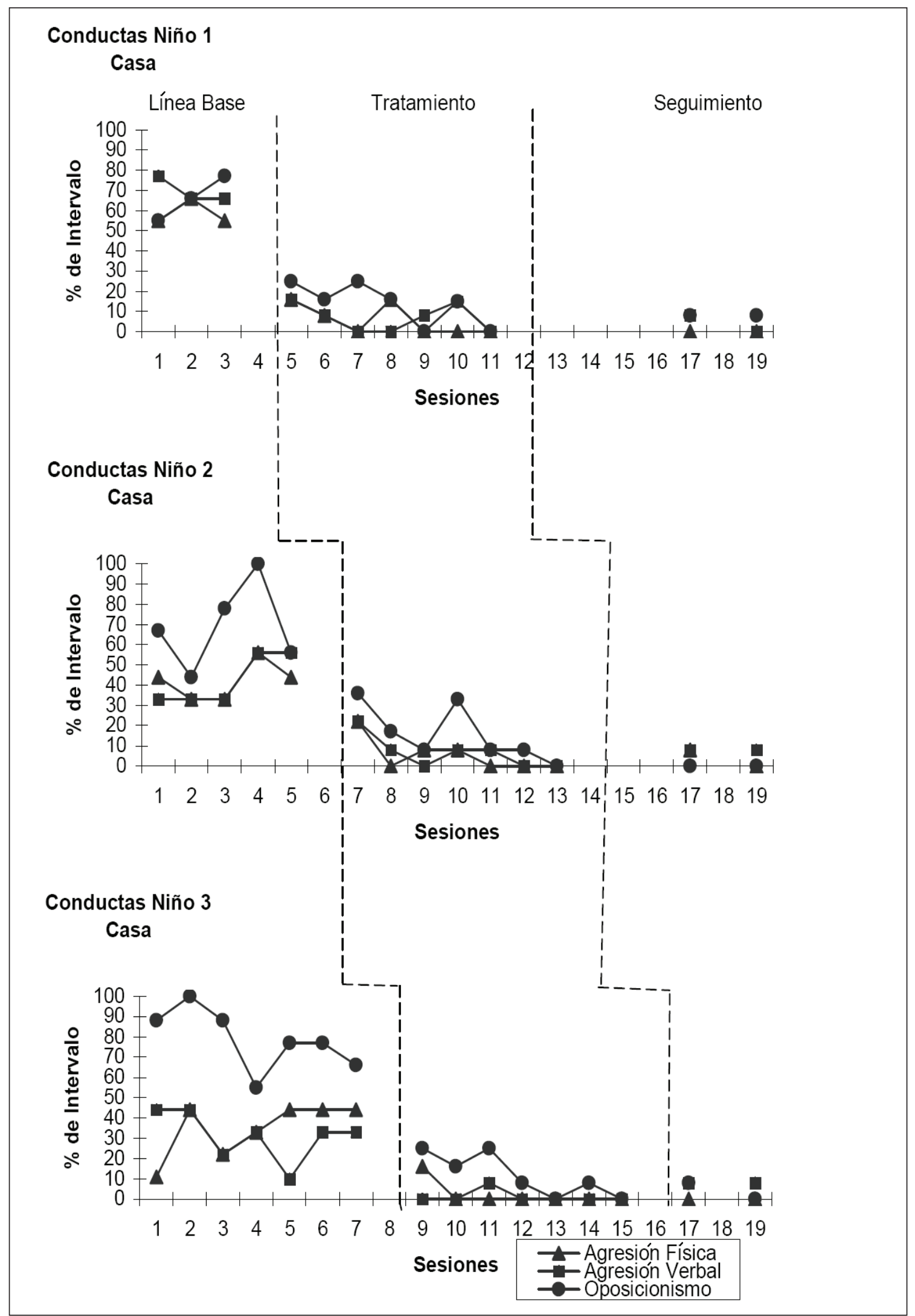

Figura 8: Cambios Conductuales en los niños en contexto familiar 
diferencia estadísticamente significativa $(\mathrm{p}=0,038)$ a partir de la sesión 2 de tratamiento.

En el caso de las instrucciones y contingencias aplicadas por las profesoras en el contexto escolar, se observa un aumento significativo entre las condiciones línea base $\mathrm{y}$ tratamiento. En el caso de las instrucciones el valor $\mathrm{F}=$ $234,941(p=0,000)$, tamaño del efecto observado de 0,892 , potencia de prueba de 0,998 y contraste de varianzas de Levene no significativo $(p=0,770)$. A su vez, tanto para las condiciones de línea base y tratamiento existe ajuste a la distribución normal con $\mathrm{Z}$ de Kolmogorov Smirnov de $0,501(p=0,963)$ y de $0,626(p=0,828)$ respectivamente. El contraste post Hoc DMS evidencia un valor significativo ( $p$ $=0,017)$ a partir de la sesión 3 de tratamiento. Sobre la correcta aplicación de contingencias se obtiene un valor $\mathrm{F}=26,798$ ( $p=0,000$ ), tamaño del efecto de 0,931 y potencia de prueba de 1. Por otro lado, el contraste de varianzas de Levene no es estadísticamente significativo $(p=0,643)$ y, tanto para las condiciones de línea base y tratamiento, existe ajuste a la distribución normal con $\mathrm{Z}$ de Kolmogorov Smirnov de $0,430(p=0,993)$ y de $0,380(p=0,999)$ respectivamente. Se evidencia un valor significativo $(p=0,030)$ a partir de la sesión 3 de tratamiento, según el contraste post Hoc DMS.

A nivel de los efectos del tratamiento en la conducta agresiva verbal, física y oposicionista de los niños en el contexto escolar, se observa una disminución significativa entre la línea base y el tratamiento. En el caso de la conducta agresiva verbal se obtiene un valor $F=20,939(p=0,045)$, un tamaño del efecto de 0,913 , potencia de prueba de 0,658 y el contraste de varianzas de Levene no es significativo $(p=0,345)$. A su vez, tanto para las condiciones de línea base y tratamiento existe ajuste a la distribución normal con Z de Kolmogorov Smirnov de 0,336 $(\mathrm{p}=0,996)$ y de $0,412(p=0,928)$ respectivamente. Habiéndose realizado contraste post Hoc DMS, se evidencia un valor estadísticamente significativo $(\mathrm{p}=0,026)$ a partir de la sesión 4 de tratamiento. En el caso de la agresión física de los niños en el contexto escolar se obtiene un valor $\mathrm{F}=13,710(\mathrm{p}=$ 0,000 ), tamaño del efecto de 0,873 y la potencia de prueba es de 1 . Por otro lado, el contraste de varianzas de Levene no es estadísticamente significativo $(p=0,381) y$, tanto para las condiciones de línea base y tratamiento existe ajuste a la distribución normal con $\mathrm{Z}$ de Kolmogorov Smirnov de $0,663(p=0,772)$ y de $0,638(p=0,810)$ respectivamente. El contraste post Hoc DMS evidencia un valor estadísticamente significativo $(p=0,036)$ a partir de la sesión 5 de tratamiento. En cuanto al oposicionismo de los niños en el contexto escolar se obtiene un valor $\mathrm{F}=16,554(\mathrm{p}=0,000)$. A su vez, el tamaño del efecto es de 0,999 , la potencia de prueba es de $1 \mathrm{y}$ el contraste de varianzas de Levene no es estadísticamente significativo $(p=0,423)$. A su vez, tanto para las condiciones de línea base y tratamiento existe ajuste a la distribución normal con $\mathrm{Z}$ de Kolmogorov Smirnov de $0,373(p=0,996)$ y de $0,369(p=0,992)$ respectivamente. Se observa cambio estadísticamente significativo $(p=0,018)$ a partir de la sesión 2 de tratamiento, según el contraste post Hoc DMS.

En el contexto familiar, se observa una disminución estadísticamente significativa en las creencias irracionales de las madres entre línea base y tratamiento. Para el tremendismo, se obtiene un valor $F=19,878(p=0,000)$, un tamaño del efecto de 0,909 , la potencia de prueba es de 1 y el contraste de varianzas de Levene no significativo $(p=0,629)$. A su vez, tanto para las condiciones de línea base y tratamiento existe un ajuste a la distribución normal con $\mathrm{Z}$ de Kolmogorov Smirnov de $0,533(p=0,939)$ y de $0,667(p=0,766)$ respectivamente. El contraste post Hoc DMS indica un valor estadísticamente significativo $(\mathrm{p}=0,015)$ a partir de la sesión 3 de tratamiento. Para la autocondena de las madres se obtiene un valor $\mathrm{F}=12,059(\mathrm{p}=0,000)$, tamaño del efecto de 0,851 , potencia de prueba de 1 , contraste de varianzas de Levene no significativo $(p=0,567)$ y un ajuste normal de las condiciones de línea base y tratamiento con $\mathrm{Z}$ de Kolmogorov Smirnov de $0,651(p=0,791)$ y de $0,667(p=0,766)$ respectivamente. El contraste post Hoc DMS indica un cambio significativo (p $=0,001)$ a partir de la sesión 4 de tratamiento. En el caso de nosoportitis se obtiene un $F=16,786(p=0,001)$, un tamaño del efecto de 0,894 , una potencia de prueba de 1 , un contraste de varianzas de Levene no significativo $(p=0,395)$ y un ajuste normal de las condiciones de línea base y tratamiento $(\mathrm{Z}$ de Kolmogorov Smirnov de 0,667, p = 0,766 en ambos casos). Después de realizar contrastes post Hoc DMS, se evidencia una diferencia estadísticamente significativa $(p=0,020)$ a partir de la sesión 3 de tratamiento.

En el caso de las instrucciones y contingencias de refuerzo y castigo aplicadas por las madres en el contexto familiar, se observa un aumento significativo entre las condiciones línea base y tratamiento. En el caso de las instrucciones el valor $\mathrm{F}$ obtenido es de 17,793 ( $\mathrm{p}=0,000)$, el tamaño del efecto observado de 0,899 , la potencia de prueba es de 1 y el contraste de varianzas de Levene no es significativo ( $p$ $=0,389$ ). A su vez, tanto para las condiciones de línea base $\mathrm{y}$ tratamiento existe ajuste a la distribución normal con $\mathrm{Z}$ de Kolmogorov Smirnov de 0,534 $(p=0,938)$ y de 0,541 $(p=0,932)$ respectivamente. El contraste post Hoc DMS evidencia un valor significativo $(p=0,044)$ a partir de la sesión 4 de tratamiento. Sobre la correcta aplicación de contingencias se obtiene un valor $\mathrm{F}=22,713(\mathrm{p}=0,000)$, tamaño del efecto de 0,919 y potencia de prueba de 0,896 . Por otro lado, el contraste de varianzas de Levene no es estadísticamente significativo $(p=0,147) y$, tanto para las condiciones de línea base y tratamiento existe ajuste a la distribución normal con $\mathrm{Z}$ de Kolmogorov Smirnov de $0,550(\mathrm{p}=0,922)$ y de $0,374(\mathrm{p}=0,999)$ respectivamente. Se evidencia un valor significativo $(\mathrm{p}=0,003)$ a partir de la sesión 1 de tratamiento, según el contraste post Hoc DMS.

A nivel de los efectos del tratamiento en la conducta agresiva verbal, física y oposicionista de los niños en el 
contexto familiar, se observa una disminución significativa entre la línea base y el tratamiento. En el caso de la conducta agresiva verbal se obtiene un valor $\mathrm{F}=9,628(\mathrm{p}=0,000)$, un tamaño del efecto de 0,828 , la potencia de prueba de 1 y el contraste de varianzas de Levene no es significativo $(\mathrm{p}=0,443)$. A su vez, tanto para las condiciones de línea base y tratamiento existe ajuste a la distribución normal con $\mathrm{Z}$ de Kolmogorov Smirnov de 0,511 $(\mathrm{p}=0,957)$ y de $0,653(p=0,787)$ respectivamente. Habiéndose realizado contraste post Hoc DMS, se evidencia un valor estadísticamente significativo $(p=0,033)$ a partir de la sesión 5 de tratamiento. En el caso de la agresión física de los niños en el contexto familiar se obtiene un valor $\mathrm{F}=19,280(\mathrm{p}=$ 0,001 ), tamaño del efecto de 0,906 y la potencia de prueba es de 0,767 . Por otro lado, el contraste de varianzas de Levene no es estadísticamente significativo $(p=0,824)$ $\mathrm{y}$, tanto para las condiciones de línea base y tratamiento existe ajuste a la distribución normal con $\mathrm{Z}$ de Kolmogorov Smirnov de $0,461(p=0,983)$ y de $0,621(p=0,836)$ respectivamente. El contraste post Hoc DMS evidencia un valor estadísticamente significativo $(p=0,000)$ a partir de la sesión 5 de tratamiento. En cuanto al oposicionismo de los niños en el contexto familiar se obtiene un valor $\mathrm{F}$ $=18,050(\mathrm{p}=0,033)$. A su vez, el tamaño del efecto es de 0,900 , la potencia de prueba es de 0,744 y el contraste de varianzas de Levene no es estadísticamente significativo (p $=0,392$ ). A su vez, tanto para las condiciones de línea base y tratamiento existe ajuste a la distribución normal con $Z$ de Kolmogorov Smirnov de 0, $517(\mathrm{p}=0,952)$ y de 0, 322 $(p=1,000)$ respectivamente. Se observa cambio estadísticamente significativo $(\mathrm{p}=0,016)$ a partir de la sesión $2 \mathrm{de}$ tratamiento, según el contraste post Hoc DMS.

\section{Análisis cualitativo de los cambios.}

En el contexto escolar las profesoras señalaron un aumento en su sensación de autoeficacia como educadoras y un aumento de su motivación por trabajar con los niños. Además señalaron que los niños que participaron del estudio experimentaron un notable cambio conductual que generó una mejora en el clima social de la sala de clases, y un aumento de su rendimiento.

En el contexto familiar las madres señalaron sentirse con más herramientas para tratar a sus hijos. Además reportaron sentir más emociones positivas en relación a sus hijos y una mejora de las relaciones al interior de la familia. Las madres reportaron realizar mayores actividades con los niños fuera del hogar (ej. ir con los niños a casas de amigos y familiares) que antes de la intervención no realizaban debido a la mala conducta de los niños. Finalmente reportan un cese de las prácticas de maltrato hacia los niños.

Por otro lado, se evidenció una mejora en la relación y una mayor coordinación entre las docentes y las madres.

Por su parte, los niños manifestaron mayor motivación a asistir al colegio y a participar de actividades familiares.
Por último, los niños aumentaron la frecuencia de comportamientos incompatibles con la agresividad para resolver sus conflictos.

\section{Discusión}

El programa de intervención fue exitoso ya que, tal como se esperaba, las madres y profesoras disminuyeron la frecuencia de las creencias irracionales asociadas a su relación con los niños. Además, mejoraron su desempeño en lo referido al manejo conductual de los niños, lo que coincidió con la disminución de la frecuencia de conductas agresivas verbales, físicas y oposicionistas en los preescolares. Los cambios favorables en las cogniciones de madres y profesoras, y en las conductas de niños, madres y profesoras han demostrado ser estadísticamente y clínicamente significativos.

Se considera que el éxito de esta intervención está dado por varios factores:

Primero, el haber trabajado en el hogar y en el colegio de los niños, lo que refuerza el planteamiento de Alberto \& Troutman (1999) referido a la necesidad de realizar un trabajo integral y en el contexto natural donde las conductas meta deben ser implementadas. Segundo, el haber incluido a madres y profesoras desde el primer momento para que sean ellas las que ejecuten los procedimientos de modificación conductual a los niños, ya que de este modo se interviene sobre la pauta de refuerzo mutuo entre los niños y los adultos a cargo de su formación. Esto confirma los planteamientos de Corsi, Barrera, Flores, Perivancich \& Guerra (2009), Rey (2006) y Trautmann (2008), sobre la utilidad del entrenamiento a padres y profesores. Tercero, el haber realizado el entrenamiento a las madres y profesoras incorporando tanto la dimensión cognitiva como la conductual ya que, como plantea el modelo de terapia cognitivo-conductual, los cambios en el comportamiento muchas veces deben estar asociados a un cambio en la forma de interpretar las situaciones (Ellis 1999, Ellis \& Lega 1993). Y en cuarto lugar, la planificación de la generalización de los resultados, donde en un comienzo se utilizó un programa de refuerzo continuo que gradualmente se fue transformando en un programa de refuerzo de razón variable, más resistente a la extinción.

Sobre la base de lo señalado se concluye que las intervenciones cognitivo-conductuales, como la descrita en esa investigación, se constituyen como una alternativa viable para la regulación de la agresividad entre preescolares. No obstante, es necesario destacar algunas limitaciones del estudio. En primer lugar, dado el número reducido de participantes, los resultados no pueden generalizarse al resto de la población de preescolares. Segundo, hay que señalar que el éxito de esta intervención se debe en gran medida al esfuerzo coordinado de diferentes agentes (directivos del establecimiento, profesoras, madres e investigadores), situación que en la gran mayoría de los casos es difícil de lograr. 


\section{Referencias}

Aguilar, C. \& Navarro, J. (2008). Análisis funcional e intervención con economía de fichas y contrato de contingencias en tres casos de conducta disruptiva en el entorno escolar. Revista Latinoamericana de psicología 40, 133- 139.

Alberto, P. \& Troutman, A. (1999). Applied behavior analysis for teachers. Londres: Prentice Hall.

Benítez, J.L. \& Justicia, F. (2006). El maltrato entre iguales: Descripción y análisis del fenómeno. Revista electrónica de Investigación Educativa 9, 151-170.

Caballo, V. (1998). Manual de técnicas de terapia y modificación de conducta. Madrid: Siglo XXI de España Editores.

Campaña, M.A., Fredes, V. \& Gutiérrez, L. (2008). Diseño de una escala para medir creencias irracionales asociadas al rol de madre y profesora. Manuscrito no publicado, Universidad del Mar, Chile.

Cooper, J. O., Heron, T. E. \& Heward, W. L. (2006). Applied behavior analysis. Second Edition. New York: Prentice Hall.

Corsi, E., Barrera, P., Flores, C., Perivancich, X. \& Guerra, C. (2009). Efectos de un programa combinado de técnicas de modificación conductual para la disminución de la conducta disruptiva y el aumento de la conducta prosocial en escolares chilenos. Acta Colombiana de Psicología 12, 67-76.

Corsi, E., Guerra, C. \& Plaza, H. (2007). Diseño, implementación y evaluación de un programa de manejo conductual para padres de niños con Síndrome de Asperger. Psicología Conductual,15, 253 - 266.

De la Barra, F., Toledo, V. \& Rodríguez, J. (2003). Estudio de salud mental en dos cohortes de niños escolares de Santiago Occidente: Predictores tempranos de problemas conductuales y cognitivos. Revista Chilena de Neuropsiquiatría, 41, 65-76.

Ellis, A. (1999). Una terapia breve más profunda y duradera. Barcelona: Paidós.

Ellis, A. \& Lega, L. (1993). Cómo aplicar algunas reglas básicas del método científico al cambio de las ideas irracionales sobre uno mismo, otras personas y la vida en general. Psicología Conductual, 1, $101-110$.

Forehand, R. \& Mc Mahon, R. (1981). A clinician's guide to parent training. New York: Guilford Press.

Foxx, R. (1982a). Increasing behaviors of severely retarded and autistic persons. Illinois: Research Press.

Foxx, R. (1982b). Decreasing behaviors of persons with severe retardation and autism. Illinois: Research Press.
Gutiérrez, H., Barrios, A., de Dios, M.J., Montero, I. \& del Barrio, C. (2008). The incident of peer bullying as multiple maltreatment among Spanish secondary school students. International Journal of Psychology and Psychological Therapy 8, 247-257.

Kellam, S., Rebok, G., Ialongo, N. \& Mayer, L. (1994). The course and malleability of aggressive behavior from early first grade into middle school: Results of a developmental epidemiologically based preventive trial. Journal of Child Psychology and Psychiatry, 35, 259- 281.

Livacic-Rojas, P., Espinoza, M. \& Ugalde, F. (2004). Intervenciones psicológicas basadas en la evidencia para la prevención de la delincuencia juvenil. Un programa de habilidades sociales en ambientes educativos. Terapia Psicológica, 22, 83-91.

Lozano, L. \& García, E. (2000). El rendimiento escolar y los trastornos emocionales y comportamentales. Psicothema 12, 340- 343.

Mestre, M.V., Tur, A.M., Samper, P., Nácher, J.M. \& Cortés, M.T. (2007). Estilos de crianza en la adolescencia y su relación con el comportamiento prosocial. Revista latinoamericana de psicología, 39, 211-225.

Nansel, T.R., Craig, W., Overpeck, M.D., Saluda, G. \& Ruan, J. (2004). Cross-national consistency in the relationship between bullying behaviors and psychosocial adjustment. Archives of Pediatrics and Adolescent Medicine 158, 730-736.

Ortega, R. \& Monks, C. (2005). Agresividad injustificada entre preescolares. Psicothema, 17, 453-458.

Pérez, V., Fernández, A. M., Rodríguez, J. \& De La Barra, F. (2005). Evaluación del efecto de una intervención conductual en la agresividad escolar. Terapia Psicológica, 23, 91-98.

Rey, C. (2006). Entrenamiento de Padres: Una revisión de sus principales componentes y aplicaciones. Revista Infancia, Adolescencia y Familia, $1,61-84$.

Schwartz, D., Dodge, K., Pettit, G. \& Bates, J. (1997). The early socialization of aggressive victims of bullying. Child Development 68, 665-675.

Trautmann, A. (2008). Maltrato entre pares o "Bullying": Una visión actual. Revista Chilena de Pediatría, 79, 13-20.

Trianes, M.V. \& Fernández-Figarés, C. (2001). Aprender a ser persona y a convivir: Un programa para secundaria. Bilbao: Descleé de Broker.

Urzúa, A., Ramos, M., Alday, C. \& Alquinta, A. (2010). Madurez neuropsicológica en preescolares: Propiedades psicométricas del test CUMANIN. Terapia Psicológica, 28, 13-25.

Zerón, A. M. (2002). Violencia Juvenil en el espacio escolar. Estudio sociológico comprensivo de la experiencia escolar. Tesis no publicada para obtener el grado de Doctora en Ciencias de la Educación, Pontificia Universidad Católica de Chile. 